\title{
Monolithically Integrated Tunable Mode-Locked Laser Diode Source with Individual Pulse Selection and Post- Amplification
}

\author{
X. GUo ${ }^{1, *}$,A. H. QuARTeRman ${ }^{1,2}$, A. WOnfor ${ }^{1}$, R. V. Penty ${ }^{1}$, AND I. H. White ${ }^{1}$ \\ ${ }^{1}$ Centre for Photonic Systems, Department of Engineering, University of Cambridge, Cambridge, CB3 OFA, UK \\ 2 now at School of Science and Engineering, University of Dundee, Dundee, DD1 4HN, UK \\ *Corresponding author:xg218@cam.ac.uk
}

Received XX Month XXXX; revised XX Month, XXXX; accepted XX Month XXXX; posted XX Month XXXX (Doc. ID XXXXX); published XX Month XXXX

\begin{abstract}
We report the generation of high peak-power picosecond optical pulses in the $1.55 \mu \mathrm{m}$ spectral band from a monolithically mode-locked laser integrated with a pulse-selector and power booster. High peak power $(>1$ W) pulses with durations of $15.4 \mathrm{ps}$ at a $55 \mathrm{MHz}$ selected rate are demonstrated, indicating that this device shows promise as a high peak power pulsed light source for biophotonic applications.
\end{abstract}

\section{(C) 2016 Optical Society of America}

OCIS codes: (140.4050) Mode-locked lasers; (060.5060) Phase modulation; (250.5980) Semiconductor optical amplifiers

http://dx.doi.org/10.1364/OL.99.099999

Monolithic modelocked laser diodes (MLLDs) have been extensively studied for many years, demonstrating a broad range of attractive characteristics such as small size, stability, the potential for low fabrication costs when in mass production, direct electrical driving, and wide potential operating wavelengths range. MMLLDs offer excellent opportunities for numerous applications, including optical communications [1], optical sampling [2], clock distribution [3], and fiber radio [4].

However the pulse durations and corresponding peak powers that can be achieved by single chip monolithic devices have been limited, as stable pulse build up occurs near to the threshold current, so as to avoid saturation issues that otherwise prevent modelocking. In recent years the generation of high power short pulses with much lower repetition rates, down to the $\mathrm{MHz}$ range, has become more desirable for emerging applications, such as multi-photon imaging [5], optical coherence tomography [6], asynchronous optical sampling [7], sensing systems [8] and micromachining [9]. As a result, attention has been given to developing MMLLDs with higher power and reduced repetition rates. Increased peak powers have been achieved by using either master oscillator power amplifier (MOPA) or tapered laser diode configurations. Koch et al. [10] demonstrated the first monolithically integrated hybrid modelocked quantum well (QW) MOPA device for optical clock recovery applications, with a pulsewidth of $6.3 \mathrm{ps}$, output power of $8.3 \mathrm{dBm}$ and extinction ratio (ER) over $12 \mathrm{~dB}$. Thompson et al. [11] reported a pulse duration of $780 \mathrm{fs}$, with a 500 $\mathrm{mW}$ peak power in a quantum dot (QD) modelocked tapered device with $4 \mu \mathrm{m}$ width absorber waveguide and a $1.46 \mathrm{~mm}$ long and $3.6^{\circ}$ flared gain region. The high repetition rates of MMLLDs, due to their inherently short cavities, have also been addressed monolithically. For example, Cheung et al. [12] have demonstrated the emission of $36 \mathrm{ps}$ pulses at $1 \mathrm{GHz}$ from a $41 \mathrm{~mm}$ long device with integrated active QW and passive waveguide structures. Latkowski et al. [13] have demonstrated a mode-locked extended cavity QW ring laser at 1.58 $\mu \mathrm{m}$ wavelength with a repetition rate of $2.5 \mathrm{GHz}$ in the form of a photonic integrated circuit (PIC) with a $33 \mathrm{~mm}$ long cavity. Of course, high peak powers and low repetition rates can be realized at the same time in non-monolithic configurations, but only with increased cost and complexity compared with monolithic devices. For example, Ding et al. [14] have reported picosecond optical pulses with power of 208.2 $\mathrm{mW}$, pulse energy of $321 \mathrm{pJ}$, and peak power of $30.3 \mathrm{~W}$ at $648 \mathrm{MHz}$ from a QD MLLD and an external tapered QD semiconductor optical amplifier (SOA).

MMLLDs can also achieve both high peak power and low repetition rates simultaneously by integrating different components on a single chip. However, their widespread adoption has been hindered by the prohibitive cost of designing bespoke components and accessing foundry facilities. One feasible solution would be to take a similar development path to that followed by microelectronic integration technology, that is, to provide a basic set of generic standardized components, from which more complex circuits can be built. Recently, indium phosphide (InP) generic foundries have used a selection of basic optoelectronic components to assemble application specific photonic integrated circuits (ASPICs) [15]. In this work therefore a MMLLD is constructed using a generic approach for InP-based PICs enabled by the EU FP7 PARADIGM project, the fabrication being performed by an external foundry [16]. The design comprises predefined standard building blocks including: SOA, Mach-Zehnder 
modulator (MZM), distributed Bragg reflector (DBR), and passive waveguides.

This generic foundry design and fabrication scheme has been reported in our previous work [17]. Here we showed a proof-ofprinciple monolithically integrated MMLLD with integrated pulse selector and amplifier where bursts of high power double pulses are delivered at pre-set repetition rates. In this work we describe a similar concept but with an enhanced design of a longer master oscillator cavity to achieve a lower fundamental repetition rate. Improved fabrication of the active-passive interfaces greatly decreases the buttjoint losses and reflections, hence allowing higher current injection into the power amplifier, and in turn enabling higher energy pulses [16]. Individual pulse selection of $15.4 \mathrm{ps}$ at a $55 \mathrm{MHz}$ repetition rate has been demonstrated, with high peak powers exceeding $1 \mathrm{~W}$. Such a rate is to the best of our knowledge the lowest demonstrated so far from a QW-based InP monolithically integrated laser diode based system.

One general physical approach to achieve lower repetition rate ultrashort pulses generation in modelocked lasers is based on the elongation of the laser cavity. The fundamental repetition rate of a modelocked laser is inversely proportional to its resonator length, hence using longer cavities leads to lower pulse repetition rate and consequently higher pulse energy assuming that the average output power is constant. The following simple relationship in Eq. (1) describes this design principle:

$$
\mathrm{E}_{p} \propto P_{a v} T_{r} \propto P_{a v} \frac{\eta L}{c}
$$

where $\mathrm{E}_{p}$ is the pulse energy of the modelocked laser, $P_{a v}$ is the pulse average power, $T_{r}$ is the cavity round trip time, $\eta$ is the refractive index of the cavity medium, $L$ is the cavity length, and $c$ is the speed of light. However the demonstration of high peak power at a repetition rate lower than $5 \mathrm{GHz}$ in all active cavity configurations becomes rather problematic as the long active region requires strong electrical pumping. Therefore it is more feasible to use active-passive monolithic integration technology. In this design the long cavity is realized by confining the gain and absorber regions in one short section of the cavity while the rest is a long passive waveguide with low loss waveguide transition regions (1-1.5 dB).
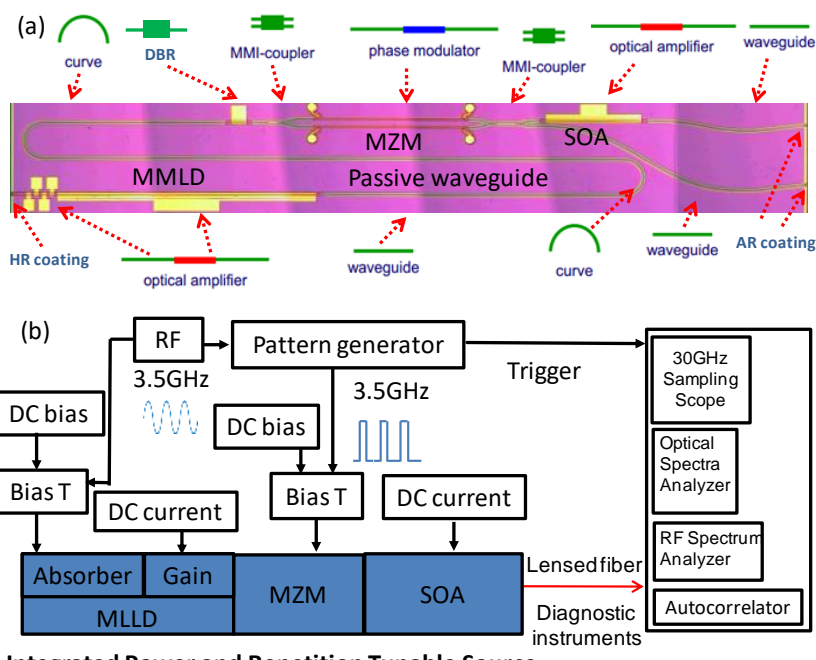

Integrated Power and Repetition Tunable Source

Fig. 1: (a) Microscope photograph of the device and (b) experimental set up

A microscope photograph of the MMLLD and associated driving electronics is shown in Fig. 1. This design features an $11 \mathrm{~mm}$ cavity length making possible a low fundamental mode-locked repetition rate of $3.55 \mathrm{GHz}$. The passive waveguide has a loss of $\sim 8 \mathrm{~dB} / \mathrm{cm}$. Care is taken at the active-passive interfaces to minimize butt-joint losses and back reflections which would otherwise limit the allowed gain in the optical amplifier sections as well as cause coupled-cavity effects. To fit onto the allocated $6 \times 4 \mathrm{~mm}$ sector on an MPW wafer, the cavity is folded and realised with circular arcs of $180^{\circ}$ curves and $S$ bends with $180 \mu \mathrm{m}$ radius. The output waveguide of this design is terminated with a spot size converter (SSC) building block, angled at $7^{\circ}$, to increase the off-chip coupling efficiency and reduce the back reflections. Lensed fiber to chip coupling losses of $\sim 3 \mathrm{~dB}$ have been measured. The master oscillator consists of four $50 \mu \mathrm{m}$ long saturable absorber (SA) sections, a $2 \mathrm{~mm}$ long gain section, a $8.6 \mathrm{~mm}$ long passive waveguide and a 120 $\mu \mathrm{m}$ long DBR section with grating coupling constant $\kappa$ of $50 / \mathrm{cm}$ and reflection peak wavelength of $1550 \mathrm{~nm}$ as an output coupler. The output pulses of the master oscillator pass through a MZM with two $1090 \mu \mathrm{m}$ long electro-optic phase shifters, and then controlled to send maximum power through a splitter, either to a direct output or to a different output via a $750 \mu \mathrm{m}$ long SOA. The device is mounted on a copper heatsink whose temperature is maintained at $20^{\circ} \mathrm{C}$. Light is coupled into the diagnostic equipment via a lensed fibre positioned close to the desired anti-reflection (AR) facet output. For the experiments, printed circuit boards were designed with high frequency Coplanar Waveguides (CPWs) to allow GHz-range probing to the SA and MZM sections of the devices.

Initially the master oscillator light-current characteristic and slope efficiency of the laser are measured using a broad area photodiode with all SA and gain sections connected together through passive waveguide. From Fig. 2 (a), the laser threshold current is $48 \mathrm{~mA}$ and the slope efficiency is $0.016 \mathrm{~W} / \mathrm{A}$ at the onset of lasing and decreases to $0.002 \mathrm{~W} / \mathrm{A}$ at a bias current of $200 \mathrm{~mA}$. A forward resistance value of 4 $\Omega$ is measured. The transparency current of the SOA in this device is measured to be $12.5 \mathrm{~mA}$. Fig. 2 (b) shows the output power of the SOA section for a range of different forward biases up to a maximum DC amplifier bias current of $200 \mathrm{~mA}$ with the laser driving current also being varied up to $200 \mathrm{~mA}$. It is observed that an increase in DC amplifier current causes an increase in output power and delivers an average optical power of up to $13 \mathrm{~mW}$ for a DC amplifier current equal to $200 \mathrm{~mA}$.
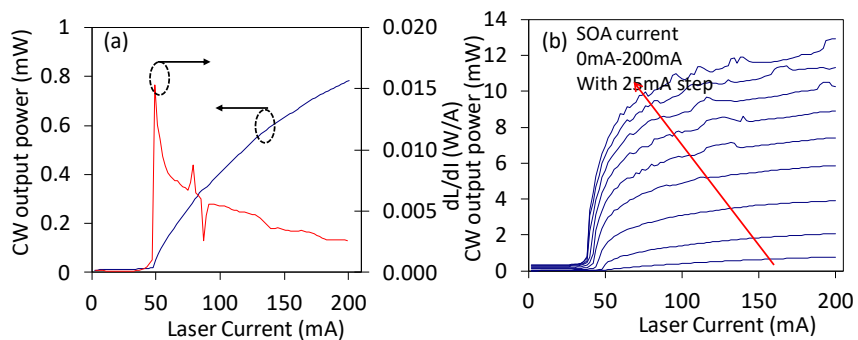

Fig. 2: (a) LI performance of the laser diode and (b) laser CW power with different SOA bias.

The MMLLD is then characterized in the passively modelocked regime. Two SA sections connected together to form a $100 \mu \mathrm{m}$ long SA with the other two SA sections connected to the gain section is found to be the best length ratio for stable passive mode-locking operation and is therefore the SA contact configuration used throughout the work described in this paper. Passive mode-locking is investigated with gain currents of $85 \mathrm{~mA}$ up to $220 \mathrm{~mA}$ and SA bias voltages of $-0.7 \mathrm{~V}$ to -1.75 $\mathrm{V}$. The lasing wavelength is close to $1555 \mathrm{~nm}$ over the whole range. Fig. 3 shows the passive mode-locking regimes with different RF signal to noise ratios (10 MHz span, $10 \mathrm{kHz}$ resolution bandwidth, $1 \mathrm{kHz}$ video bandwidth). The quality of the modelocked pulses is indicated by the narrowness of the RF spectral lines and RF SNR. The regimes with SNR higher than $35 \mathrm{dBc}$ are investigated in detail as these indicate potential passive mode-locking, and in turn could be expected to support hybrid mode-locking. 


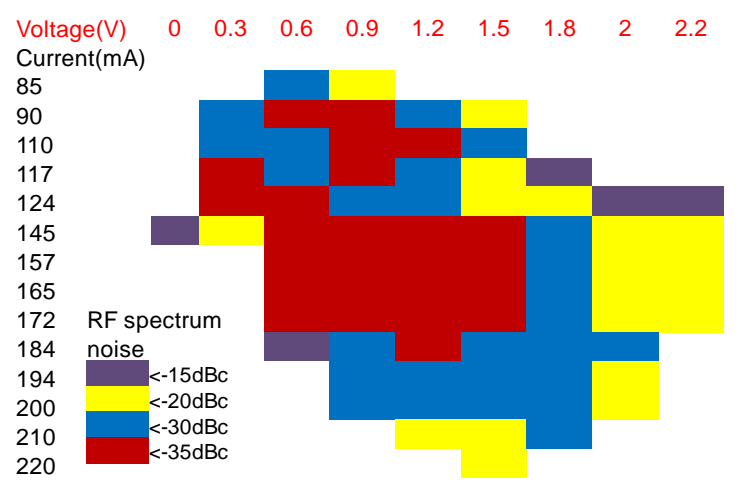

Fig. 3: Passive mode-locking quality estimation with different combinations of driving conditions.

Stable hybrid mode locking is achieved by applying a DC current to the gain section and a reverse DC bias plus an RF modulation signal to the SA via a bias tee. To investigate the detuning characteristics of the hybrid modelocked laser, the applied RF signal frequency is detuned from the laser cavity fundamental repetition rate. From Fig. 4 (a), it is observed that as the RF frequency approaches the fundamental repetition rate, the laser beat frequency is pulled to the applied signal and increases in power. The locking range increases with the RF power applied to the SA of the laser, and the synchronization with the external RF signal under hybrid mode-locking strongly decreases the lowfrequency phase noise. This is indicated by the decrease in phase noise by $9 \mathrm{~dB}$ and linewidth of the first harmonic peak in the RF spectrum, from about $476 \mathrm{kHz}$ FWHM under passive mode-locking to below 100 $\mathrm{kHz}$, limited by the external $\mathrm{RF}$ generator and $\mathrm{RF}$ analyzer which introduce jitter (Fig. 4 (a) inset). Increasing the injection current further broadens the RF linewidth and destabilizes the mode-locking. Significantly larger locking ranges could be achieved by using larger locking RF powers, though even the relatively power of $0 \mathrm{dBm}$ can guarantee hybrid mode-locking over a $6 \mathrm{MHz}$ range. Note that this locking range was achieved without requiring costly $\mathrm{RF}$ amplifiers. The relatively low signal power needed for hybrid mode-locking of this laser and the large hybrid mode-locking range indicate the excellent potential for practical applications using RF modulated optical signals.
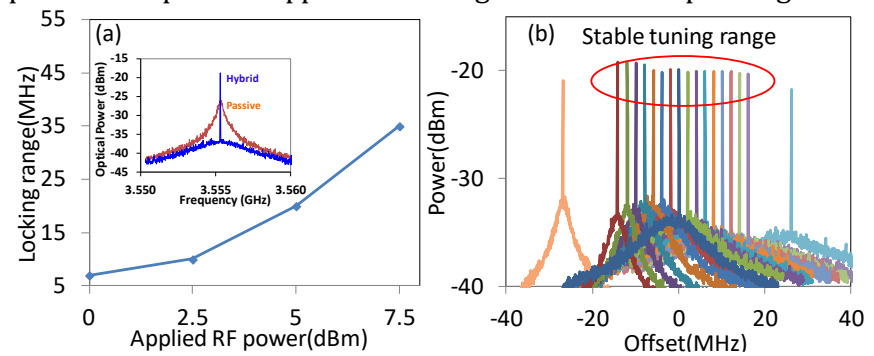

Fig. 4: RF spectra of hybrid mode-locking frequencies close to fundamental repetition rate, showing (a) the locking range as a function of the applied RF power (inset: RF spectrum of hybrid and passive modelocking) and (b) a continuous tuning range greater than $35 \mathrm{MHz}$.

The RF spectra of the signals are shown for the MLLD under hybrid mode-locking, where the operating injection current and RF driving frequency have been optimized for maximum pedestal suppression, using an RF power of $7.5 \mathrm{dBm}$ to the SA. RF spectra of the laser at several different external RF frequencies are shown in Fig. 4 (b). In this graph, $0 \mathrm{MHz}$ detuning corresponds to the point where the applied $\mathrm{RF}$ frequency is equal to the fundamental repetition rate and positive detuning occurs when higher RF frequency was applied. A measured locking range of greater than $35 \mathrm{MHz}$ at $7.5 \mathrm{dBm}$ RF applied power was achieved with an extinction ratio larger than $14 \mathrm{dBc}$. All further results reported use an SA DC bias of $-1.51 \mathrm{~V}$ with external locking RF power of $7.5 \mathrm{dBm}$ and an injection current of $97 \mathrm{~mA}$, unless otherwise noted, since these operating conditions result in the lowest phase noise.

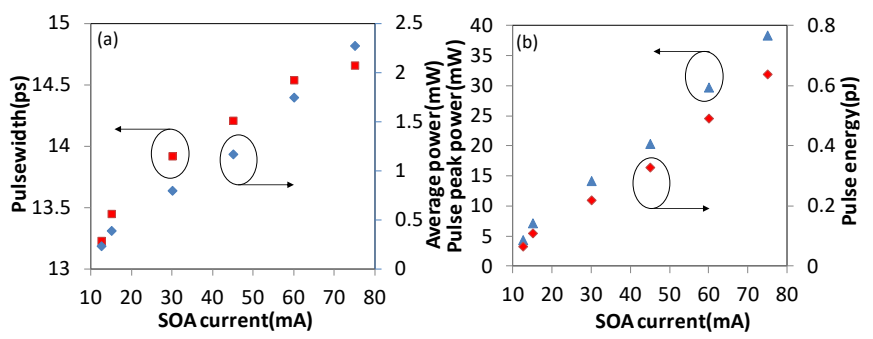

Fig. 5: Hybrid mode-locking as a function of SOA bias (a) pulsewidth and average power (b) pulse peak power and pulse energy.

The pulse output in hybrid mode locking is measured by collinear autocorrelation. Fig. 5 shows that the mode locking pulsewidth increases with increasing SOA current, due to the broadening effect of the SOA. The pulse peak power and pulse energy also increase linearly with increasing applied SOA current, with the maximum pulse peak power and energy achieved being $38 \mathrm{~mW}$ and $0.64 \mathrm{pJ}$ respectively at $75 \mathrm{~mA}$ SOA bias current.
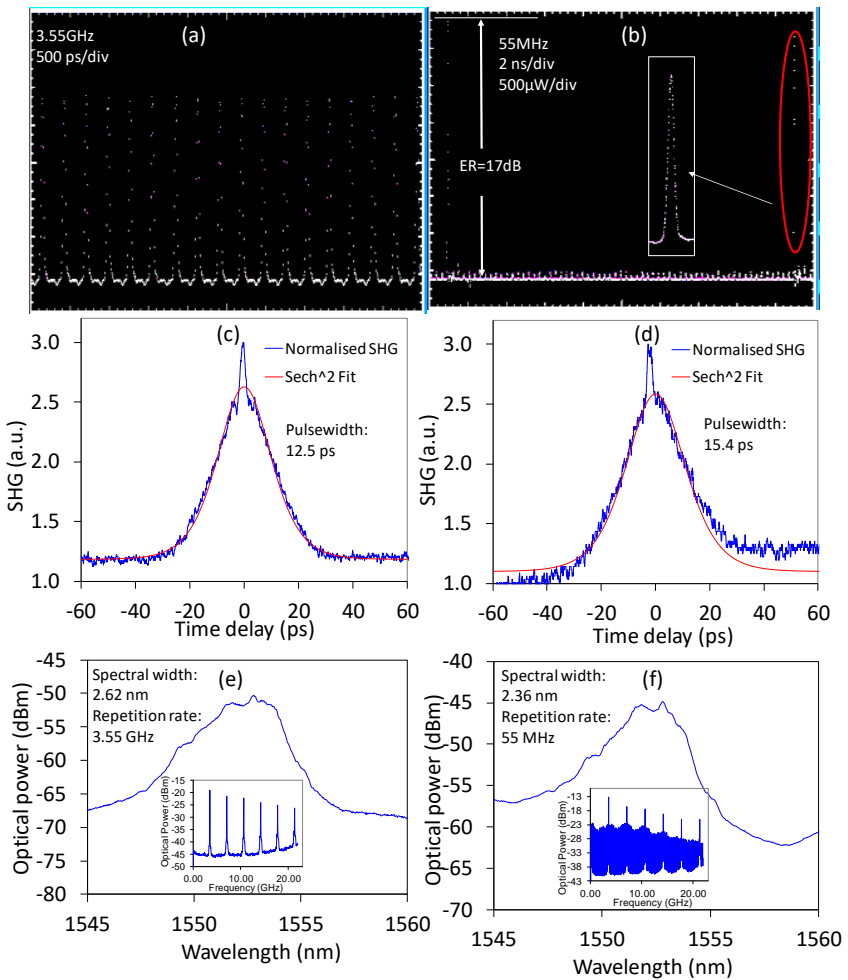

Fig. 6 (a-b) Sampling oscilloscope traces of the unmodulated $3.55 \mathrm{GHz}$ (500ps/div) and modulated $55 \mathrm{MHz}$ (2ns/div) pulse trains and corresponding (c-d) autocorrelation traces, (e-f) optical spectra and RF spectra. In (b) single pulses are visible at either side of the frame, with low power pulses in between due to the imperfect $(17 \mathrm{~dB})$ extinction ratio of the MZM.

Pulse picking was realized by applying a square wave switching between $-10.4 \mathrm{~V}$ and $-7.4 \mathrm{~V}$ (DC bias $-8.9 \mathrm{~V}$ with RF bias changes from $1.5 \mathrm{~V}$ to $1.5 \mathrm{~V}$ ) at different sub-harmonic frequencies of the fundamental repetition rate, and at different duty cycles to one arm of the MZM, while leaving the other arm with DC reverse bias of $-4.8 \mathrm{~V}$ to balance the null voltage. Due to the high frequency CPWs on the PCBs and the high frequency $50 \mathrm{ohms}$ matching line terminations, the electrical reflections are negligible. The neighboring optical pulses are therefore strongly suppressed. Fig. 6 (a-b) shows the oscilloscope waveforms of the unmodulated unamplified output and the modulated and amplified output at a 1:64 duty cycle, which corresponds to 
repetition rates of $3.55 \mathrm{GHz}$ and $55 \mathrm{MHz}$ respectively. The measured pulse suppression of neighbouring pulses is better than $17 \mathrm{~dB}$. Even lower repetition rates could be achieved, limited only by the electronic bandwidths.

The corresponding autocorrelations of a typical pulse train assuming a sech ${ }^{2}$ pulse profile, optical spectra and RF spectra and together with their pulse selection counterparts are shown in Fig. 6 (c-f) respectively. When no voltage is applied to either arm of the MZM, the output of the device is an unmodulated train of 12.5 ps pulses at a repetition rate of $3.55 \mathrm{GHz}$. When the SOA is operated at $90 \mathrm{~mA}$ together pulse selection by the MZM at a rate of $55 \mathrm{MHz}$, the spectral width is measured to be $2.36 \mathrm{~nm}$ and the pulse width increases to $15.4 \mathrm{ps}$ due to broadening effects in the SOA. The peak power and energy of the pulse increase from $9.6 \mathrm{~mW}$ and $0.07 \mathrm{pJ}$ to $1.1 \mathrm{~W}$ and $19.3 \mathrm{pJ}$ respectively, corresponding to $14.6 \mathrm{~dB}$ amplification.

When the pulse trains are modulated at low repetition rates by the MZM and a high drive current is applied to the amplifier section, much more efficient power conversion occurs, as the lower repletion rate allows more gain per pulse, yielding greater peak power and higher pulse energy from the SOA output facet, though the average power decreases slightly due to the reduced repetition rate. Fig. 7 shows that the pulsewidth, peak power and the pulse energy all decrease with increasing pulse repetition rate, while the average power stays approximately the same over a significant range, indicating that the SOA is operating in the saturation regime.
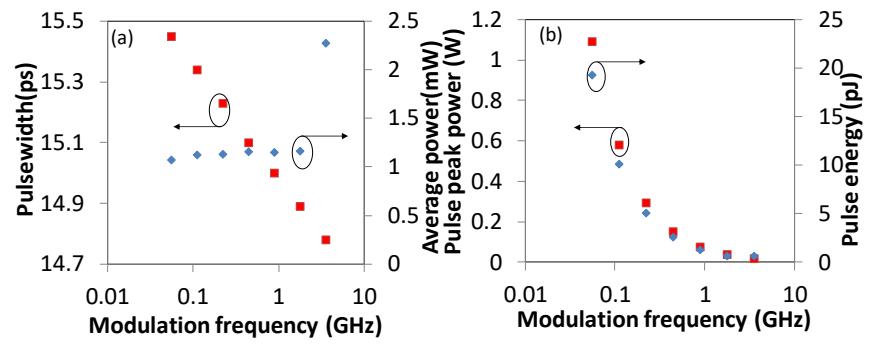

Fig.7. MLLD-modulator-SOA operation as a function of modulation frequencies: (a) pulsewidth and average power study, (b) pulse peak power and pulse energy study.

Direct electronic modulation over a large range of frequency by using a MZM plus some continuous tuning of locking range by changing the frequency of the RF voltage applied to the SA make possible both the coarse discrete switching and fine continuous control of the repetition locking range. Devices of this type are thus potentially suitable as a low-cost source for applications such as asynchronous optical sampling (ASOPS) [18].

Table 1. Pulse performance comparison

\begin{tabular}{llll}
\hline Pulse parameters & $(1)$ & (2) & (3) \\
\hline Spectral width (nm) & 2.62 & 2.47 & 2.36 \\
Pulsewidth (ps) & 13 & 14.8 & 15.4 \\
Time-bandwidth product & 4.2 & 4.54 & 4.53 \\
Max. pulse energy (pJ) & 0.07 & 0.63 & 19.3 \\
Max. peak power (mW) & 9.57 & 38.1 & 1100 \\
Min. rep rate (GHz) & 3.55 & 3.55 & 0.055 \\
\hline
\end{tabular}

The measured performance for (1) pure hybrid modelocking, (2) hybrid modelocking through the SOA output biased at $90 \mathrm{~mA}$ current, and (3) hybrid modelocking with pulse selection at a repetition rate of $55 \mathrm{MHz}$ and with a SOA current of $90 \mathrm{~mA}$ are compared in Table 1. It can be seen that the peak power increases by $6 \mathrm{~dB}$ with just SOA amplification and $20.6 \mathrm{~dB}$ at the $64 \times$ reduced repetition rate without major pulse performance degradation, greatly increasing the input current to output peak power conversion efficiency.

In summary, we demonstrate an enhanced monolithically integrated mode-locked laser diode source constructed in a generic foundry. A modulated pulse train is demonstrated which with pulse selection allows the generation of a $55 \mathrm{MHz}$ train of 15.4 ps pulses with peak powers exceeding $1 \mathrm{~W}$. This may be promising for applications in biophotonics which require higher optical powers and $\mathrm{MHz}$ range repetition rate than conventional laser diode short pulse sources are able to provide.

Funding. This work is funded by the UK EPSRC MUSIC project and the FP7 NMP Project PARADIGM.

\section{References}

1. R. Kaiser and B. Huttl, IEEE J. Sel. Top. Quantum Electron. 13, 125 (2007).

2. K. J. Weingarten, M. J. W. Rodwel, and D. M. Bloom, IEEE J. Quantum Electron. 24, 198 (1988).

3. P. J. Delfyett, D. H. Hartman, and S. Z. Ahmad, J. Lightwave Technol. 9, 1646 (1991).

4. A. J. C. Vieira, P. R. Herczfeld, A. Rosen, M. Ermold, E. E. Funk, W. D. Jemison, and K. J. Williams, IEEE Trans. Microwave Theor. Tech. 49, 1882 (2001).

5. M. Kuramoto, N. Kitajima, H. Guo, Y. Furushima, M. Ikeda, and H. Yokoyama, Opt. Lett., 32, 2726, (2007).

6. U. Sharma, E. W. Chang, and S. H. Yun, Opt. Express, 16, 19712, (2008).

7. P. A. Elzinga, R. J. Kneisler, F. E. Lytle, Y. Jiang, G. B. King, and N. M. Laurendeau, Appl. Opt., 26, 4303 (1987).

8. C. T. A. Brown, D. J. Stevenson, X. Tsampoula, C. McDougall, A. A. Lagatsky, W. Sibbett, F. J. Gunn-Moore, and K. Dholakia, J. Biophotonicsvol. 1, 183, (2008).

9. X. Liu, D. Du and G. Mourou, , IEEE J. Quantum Electron, 33, 1706 (1997)

10. B. R. Koch, J. S. Barton, M. Masanovic, Z. Hu, J. E. Bowers, and D. J. Blumenthal,. IEEE Photon. Technol. Lett. 19, 641 (2007).

11. M. G. Thompson, A. Rae, R. L. Sellin, C. Marinelli, R. V. Penty, I. H. White, A. R. Kovsh, S. S. Mikhrin, D. A. Livshits, and I. L. Krestnikov, Appl. Phys. Lett. 88, 133119 (2006).

12. S. Cheung, B. Jong-Hwa, R. P. Scott, N. K. Fontaine, F. M. Soares, Z. Xiaoping, D. M. Baney, and S. J. Ben Yoo, IEEE Photon. Technol. Lett. 22, 1793 (2010).

13. S. Latkowski, V. Moskalenko, S. Tahvili, L. Augustin, M. K. Smit, K. A. Williams, and E. A. J. M. Bente, Opt. Lett. 40, 77 (2015).

14. Y. Ding, R. Aviles-Espinosa, M. A. Cataluna, D. Nikitichev, M. Ruiz, M. Tran, Y. Robert, A. Kapsalis, H. Simos, C. Mesaritakis, T. Xu, P. Bardella, M. ossetti, I. Krestnikov, D. Livshits, I. Montrosset, D. Syvridis, M. Krakowski, P. Loza-Alvarez, and E. Rafailov, Opt. Express 20, 14308 (2012).

15. M. Smit, X. Leijtens, E. Bente, J. van der Tol, H. Ambrosius, D. Robbins, M. Wale, N. Grote, and M. Schell, Optoelectronics, IET, 5, 187 (2011).

16. PARADIGM. Available: http://www.paradigm.jeppix.eu/

17. X. Guo, V. Olle, A. Quarterman, A. Wonfor, R. V. Penty, and I. H. White, Opt. Lett. 39, 4144 (2014).

18. P. A. Elzinga, R. J. Kneisler, F. E. Lytle, Y. Jiang, G. B. King, and N. M. Laurendeau, Appl. Opt. 26, 4303 (1987). 